POS PROCEEDINGS

\title{
Landau Levels in Lattice QCD
}

\section{Falk Bruckmann*}

Universität Regensburg, Institut für Physik, Universitätstraße 31, 93053 Regensburg, Germany

E-mail: falk.bruckmannephysik.uni-regensburg.de

\section{Gergely Endrődi ${ }^{\dagger}$}

Institute for Theoretical Physics, Goethe University, Max-von-Laue-Strasse 1, 60438 Frankfurt am Main, Germany

E-mail: endrodiath.physik.uni-frankfurt.de

\section{Matteo Giordano; Sándor D. Katz}

Institute for Theoretical Physics, Eötvös University, and MTA-ELTE Lattice Gauge Theory

Research Group, Pázmány P. sétány 1/A, H-1117 Budapest, Hungary

E-mail: giordano@bodri.elte.hu, katzabodri.elte.hu

\section{Tamás G. Kovács ${ }^{\ddagger}$}

Institute for Nuclear Research of the Hungarian Academy of Sciences, Bem tér 18/c, H-4026

Debrecen, Hungary

E-mail: kgteatomki.mta.hu

\section{Ferenc Pittler}

HISKP(Theory), University of Bonn, Nussallee 14-16, D-53115,Bonn, Germany

E-mail:pittlerehiskp.uni-bonn.de

\section{Jacob Wellnhofer*}

Universität Regensburg, Institut für Physik, Universitätstraße 31, 93053 Regensburg, Germany

E-mail:Jacob. Wellnhoferephysik. uni-regensburg. de

The spectrum of the two-dimensional continuum Dirac operator in the presence of a uniform background magnetic field consists of Landau levels, which are degenerate and separated by gaps. On the lattice the Landau levels are spread out by discretization artefacts, but a remnant of their structure is clearly visible (Hofstadter butterfly). If one switches on a non-Abelian interaction, the butterfly structure will be smeared out, but the lowest Landau level (LLL) will still be separated by a gap from the rest of the spectrum. In this talk we discuss how one can define the LLL in QCD and check how well certain physical quantities are approximated by taking into account only the LLL.

34th annual International Symposium on Lattice Field Theory

24-30 July 2016

University of Southampton, UK

\footnotetext{
* Supported by the DFG (BR 2872/6-1 and BR 2872/7-1)

${ }^{\dagger}$ Supported by the DFG (Emmy Noether Programme EN 1064/2-1; and SFB/TRR 55)

$\ddagger$ Supported by OTKA under the grant OTKA-K-113034.

§Supported by the Hungarian Academy of Sciences under "Lendület" grant No. LP2011-011.

ISpeaker.
} 


\section{Introduction}

Strong magnetic fields play a crucial role in noncentral heavy-ion collisions, compact stars and in the evolution of the early Universe. For a recent review see [1]. Progress in the study of these problems requires a better understanding of the effect of an external magnetic field in Quantum Chromo Dynamics (QCD), the theory which describes the strong interaction between quarks and gluons. In QCD the external magnetic field has an impact on the dynamical chiral symmetry breaking in the vacuum of the theory. Below the QCD crossover temperature the order parameter for chiral symmetry breaking, i.e., the chiral condensate, is enhanced by the magnetic field, a phenomenon called magnetic catalysis [2]. Around the pseudo-critical temperature the chiral condensate instead decreases when the magnetic field is turned on (inverse magnetic catalysis) [3].

As the magnetic field enters the Dirac operator $\not D$, it affects the chiral condensate in two ways [4]. Since $\not D$ is the operator of interest, there is a direct, "valence" effect of the magnetic field on the observable. However, the magnetic field also influences the probability distribution of the gauge configurations through the fermionic determinant in the action, thus having a second, indirect effect on the chiral condensate. The latter we call the "sea" effect. In order to get some insight into the phenomena of magnetic catalysis and inverse magnetic catalysis, it is useful to study the valence and the sea effects separately.

The valence effect is studied by determining the spectrum of the Dirac operator at nonzero magnetic field $B$ on a typical configuration obtained at $B=0$. The result is an increase in the density of the low modes of the Dirac operator, which leads through the famous Banks-Casher relation [5] to magnetic catalysis. However, by turning on the magnetic field also in the sea, the fermionic determinant would suppress those gauge configurations which contain a larger density of small eigenvalues. Thus the sea effect drives the system towards inverse magnetic catalysis. It turns out that the valence effect dominates over the sea except around the pseudo-critical transition temperature [3]. Here the Polyakov loop effective potential is flat and a small effect of the magnetic field in the fermion determinant can significantly change expectation values [6].

Magnetic catalysis is often attributed to the linear dependence of the degeneracy of the lowest Landau level (LLL) on the strength of the magnetic field. A widely employed approximation in low-energy models, effective theories and functional approaches is to neglect higher Landau levels, see, e.g., Refs. [7, 8, 9, 10, 11, 12, 13, 14]. To assess the systematics of such approximations, here we study the issue of Landau levels on the lattice for the first time, and check directly their influence on the chiral condensate.

\section{Landau levels}

In the case of free ${ }^{1}$ quarks exposed to a uniform background magnetic field (which will always point in the $z$ direction), the spectrum of the Euclidean Dirac operator is organized in so-called Landau levels. We first examine them in $2 d$, and then proceed to the physical $(3+1) d$ case. We start with the continuum, finite volume case, and then go to the lattice. In a finite periodic box of

\footnotetext{
${ }^{1}$ In this context "free" means that the quarks do not interact with gluons.
} 

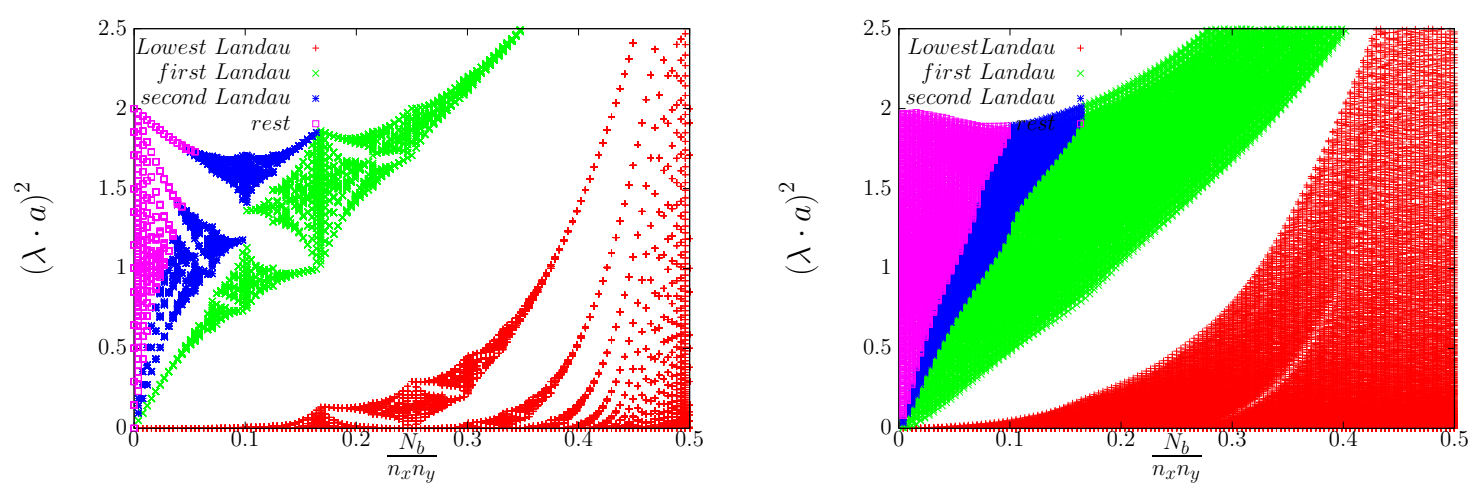

Figure 1: Classification of the lattice eigenvalues according to continuum Landau-level degeneracies. The left panel shows the spectra of the free two-dimensional Dirac operator, while the right panel shows the interacting case - evaluated on a two-dimensional slice of a typical four-dimensional gauge configuration.

area $L^{2}$, the flux of the magnetic field is quantized according to

$$
N_{b} \equiv \frac{q B L^{2}}{2 \pi} \in \mathbb{Z}
$$

and the eigenvalues of the free, massless $-\not \not^{2}$ are

$$
\lambda_{k}^{2}=|q B| k, \quad k=2 n+1-2 s_{z} \operatorname{sgn}(q B), n=0,1, \ldots, s_{z}= \pm \frac{1}{2} .
$$

The integer $k$ identifies the Landau level (LL). The degeneracy $v_{k}$ of each LL is proportional to the magnetic flux through the area of the system, i.e,

$$
v_{k}=N_{b} N_{c}\left(2-\delta_{k, 0}\right) .
$$

The separation between levels is proportional to the strength of the magnetic field. Thus, for large magnetic fields there will be a huge separation between the lowest Landau level (LLL), which (for positive $q B$ ) corresponds to $\left(n, s_{z}\right)=\left(0, \frac{1}{2}\right)$, and the rest of the spectrum, since the LLL is $B$ independent. If this separation of modes persists even after turning on the strong interaction, then one could easily explain magnetic catalysis in $2 d$ : the enhanced density of the lowest modes would in fact increase the condensate through the Banks-Casher relation.

In order to check this explanation we put the theory on a symmetric $2 d$ lattice $n_{x}=n_{y}$ and use the staggered discretization of the Dirac operator. Here and in the rest of the paper we only consider the magnetic field dependence of the operator, i.e., the valence effect, while setting $B=0$ in the fermion determinant. We use $n_{f}=2+1$ flavors of staggered quarks with physical quark masses. On the lattice the degeneracy of the LL-s is broken by lattice artefacts, and the spectrum forms a fractal structure (Hofstadter butterfly) in the $\lambda-B$ plane [15]. This is shown in the left panel of Fig. 1. The different LL-s are represented by different colors and are identified with the help of eq. (2.3) just by counting modes (and taking into account the twofold doubling of eigenvalues in two dimensions).

Next we switch on QCD interactions by taking one $2 d x-y$ slice of a typical $4 d$ QCD gauge configuration and inserting the links in the two-dimensional staggered Dirac-operator $\not_{x y}$. This 


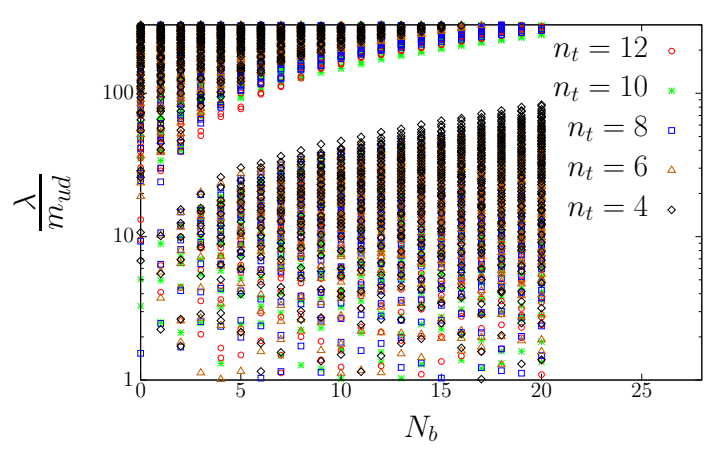

Figure 2: The $2 d$ spectrum, rescaled by the bare quark mass, as a function of the magnetic flux, for different lattice spacings, ranging from $a=0.125 \mathrm{fm}$ to $a=0.041 \mathrm{fm}$.

smears out almost entirely the butterfly structure [16] (see the right panel of Fig. 1). However, a clear gap is still present, with the number of eigenmodes below the gap exactly matching the expected continuum degeneracy (2.3). These features enable us to unambigously identify the LLL also in two dimensional QCD. The identification of the higher LL-s is however no longer possible after color interactions are included.

The reason why the LLL survives the presence of strong interactions is topological. In fact, in $2 d$ and in the continuum, the index theorem assures that the number of zero modes of $D_{x y}$ equals the magnetic flux, which is a quantized topological invariant, irrespectively of the presence of SU(3) interactions. On the lattice these become almost zero modes, which are however protected by topology and remain separated from the modes above the gap. To show that this gap is not just a lattice artefact we have to perform the continuum limit. We take lattices at five different spacings, corresponding to $4 d$ lattices with temporal extent ranging from $n_{t}=4$ up to $n_{t}=12$, keeping the physical lattice volume and temperature fixed. To make a comparison between different lattice spacings, we rescale each eigenvalue with the bare quark mass [17] and use the same physical magnetic field, which corresponds to using the same flux $\left(N_{b}\right)$ on all $n_{t}$-s. We show our results in Fig. 2. The gap between the first $3 N_{b}$ modes and the rest of the spectrum indeed persists for small flux quanta even at the smallest lattice spacing.

\section{Lowest Landau level dominance in $2 d$}

Having found a way to identify the LLL in $2 d$ QCD, we now turn our attention to its contribution to the valence effect on the chiral condensate discussed in the Introduction. We begin again with the $2 d$ case, i.e., from the study of $2 d$ slices of $4 d$ QCD configurations. The contribution from the LLL to the condensate is:

$$
\langle\bar{\psi} \psi\rangle_{B, 2 d, L L L}=\left\langle\sum_{i \in 1 \cdots 3 N_{b}} \frac{2 m}{\lambda_{i}(B)^{2}+m^{2}}\right\rangle,
$$

where $\lambda_{i}(B)$ are the eigenvalues of the finite- $B$ operator. At zero magnetic field, the contribution of the first $3 N_{b}$ modes to the condensate is

$$
\langle\bar{\psi} \psi\rangle_{B=0,2 d, L L L}=\left\langle\sum_{i \in 1 \ldots 3 N_{b}} \frac{2 m}{\lambda_{i}(0)^{2}+m^{2}}\right\rangle,
$$



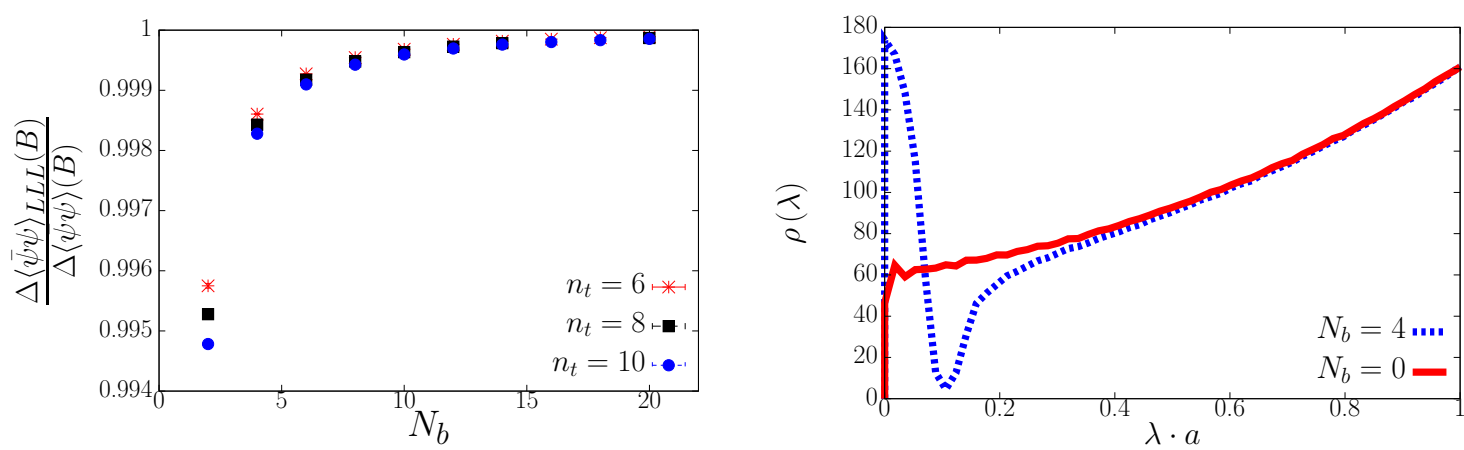

Figure 3: Left panel: the portion of the change in the condensate coming from the LLL at a fixed temperature $(T=214 \mathrm{MeV})$ as a function of the magnetic flux. Right panel: the spectral density of the $2 d$ Dirac operator at zero and non-zero external magnetic field respectively.

where $\lambda_{i}(0)$ are the eigenvalues of the zero- $B$ operator. The change in the condensate coming from the first $3 N_{b}$ modes, which at finite $B$ are precisely the LLL modes, is just the difference between (3.1) and (3.2). To quantify how much of the total change in the condensate comes from the LLL, we compute the ratio of the differences:

$$
\frac{\langle\bar{\psi} \psi\rangle_{B, 2 d, L L L}-\langle\bar{\psi} \psi\rangle_{B=0,2 d, L L L}}{\langle\bar{\psi} \psi\rangle_{B, 2 d}-\langle\bar{\psi} \psi\rangle_{B=0,2 d}}
$$

where $\langle\bar{\psi} \psi\rangle_{B, 2 d}$ and $\langle\bar{\psi} \psi\rangle_{B=0,2 d}$ are the full condensate at nonzero and zero $B$, respectively. In the left panel of Fig. 3 we show this ratio as a function of the magnetic flux for three values of the lattice spacing. Remarkably, the LLL explains almost entirely the change in the condensate. To further illustrate the LLL dominance we show the spectral density of the $2 d$ Dirac operator in the right panel of Fig. 3 for zero and non-zero magnetic field. At non-zero $N_{b}$ one can clearly see a drop in the spectral density, corresponding to the gap discussed above. Moreover, above the drop the two spectral densities are almost identical, which is the reason why the LLL dominates the change in the condensate.

\section{Landau levels in $4 d$}

After having seen how well the LLL dominance works in $2 d$, we generalize the notion of LL-s to the physically more interesting $(3+1) d$ case. Let us first discuss the eigenmodes of the free Dirac operator in a finite $4 d$ box. They factorize into an $(x, y)$ dependent part, which is one of the $2 d$ LL solutions, and into plane waves describing free propagation in the $z$ and $t$ directions. The eigenvalues of $-\not D^{2}$ are now

$$
\lambda^{2}=|q B|\left[2 n+1-2 s_{z} \operatorname{sgn}(q B)\right]+p_{z}^{2}+p_{t}^{2}, n=0,1, \ldots, s_{z}= \pm \frac{1}{2},
$$

where $p_{z}=\frac{2 \pi}{L_{z}} k_{z}$ and $p_{t}=\frac{\pi}{L_{t}}\left(2 k_{t}+1\right)$, with integer $k_{z}$ and $k_{t}$, are the momentum in the $z$ and $t$ directions, respectively. The gap, which was present in the $2 d$ case, is now filled due to the contribution of the $z$ and $t$ momenta. Thus by just looking at the spectrum we are no longer able to identify the LLL. The situation is obviously even more complicated after SU(3) interactions are 



Figure 4: Left panel: overlap between a $4 d$ mode and the $j$-th $2 d$ eigenmode, as defined in (4.2). An average over $4 d$ eigenmodes in the range $\frac{\lambda}{m_{u d}} \in[220,225]$, and an average over several gauge configurations are performed. Right panel: The fraction of the change in the valence condensate in $4 d$ that can be attributed to the change of the first $3 N_{b}$ modes on all $(z, t)$ slices as a function of the physical magnetic field. The temperature is $124 \mathrm{MeV}$.

switched on. However, we have seen that also in this case we can identify the LLL among the $2 d$ modes on an arbitrary $(z, t)$ slice. Now naturally arises the question whether these LLL $2 d$ modes have any special role in the $4 d$ spectrum. To answer this question, we begin by determining what is the overlap of a $2 d$ eigenmode with a given $4 d$ mode, i.e.,

$$
W_{j}(B)=\sum_{z, t}\left|\left(\phi_{t, z, j}(B), \psi\right)\right|^{2},
$$

where the summation is over all $(z, t)$ slices, $\phi_{t, z, j}(B)$ is the $j$-th positive eigenmode of the $2 d$ Dirac operator on the $(z, t)$ slice and $\psi$ is a $4 d$ eigenmode.

Our results are shown in the left panel of Fig. 4 for $N_{b}=8$ and $T \simeq 400 \mathrm{MeV}$. Here we averaged over modes in a small spectral window in the low part of the $4 d$ spectrum and over gauge configurations. We can see that there is a jump in $W_{j}$ after the first $3 N_{b}$ modes, which make up the LLL in the $2 d$ case. Thus the LLL gives indeed a distinct contribution to the $4 d$ modes, and it seems therefore reasonable to define the LLL contribution to the $4 d$ eigenmodes by projecting them onto the $2 d$ LLL modes.

\section{Lowest Landau level dominance in $\mathbf{4} d$}

Having defined the LLL part of a $4 d$ eigenmode we can determine the LLL contribution to the valence effect. The change in the condensate in $4 d$ due to the appearance of $3 N_{b}$ would-be zero modes on each $z, t$ slice can be calculated in a similar manner as in the $2 d$ case. The contribution to the condensate from the LLL modes at finite $B$ is identified as

$$
\langle\bar{\psi} \psi(B)\rangle_{3 N_{b}, \text { all slices }}=\left\langle\sum_{i} \frac{2 m}{\lambda_{i}(B)^{2}+m^{2}} C_{i}(B)\right\rangle,
$$

where $C_{i}(B)$ is the size of the projection of mode $i$ on the LLL subspace, $C_{i}(B)=\sum_{\text {doublers }} \sum_{j=1}^{3 N_{b}} W_{j}$, where the summation includes the negative doublers of the $2 d$ LLL modes. ${ }^{2}$ In order to calculate

\footnotetext{
${ }^{2}$ The $i$ dependence of $C$ comes from the fact that the scalar product in $W_{j}(4.2)$ has to be evaluated with $\psi_{i}$.
} 
the change in the condensate due to these modes we have to subtract the contribution of the first $3 N_{B} 2 d$ modes at $B=0$ :

$$
\langle\bar{\psi} \psi\rangle_{3 N_{b}, \text { all slices }}=\left\langle\sum_{i} \frac{2 m}{\lambda_{i}(0)^{2}+m^{2}} C_{i}^{0}\left(N_{b}\right)\right\rangle .
$$

Here $C_{i}^{0}\left(N_{b}\right)$ is the size of the projection of mode $i$ on the subspace built out of the first $3 N_{b} 2 d$ modes on each slice,

$$
C_{i}^{0}\left(N_{b}\right)=\sum_{\text {doublers }} \sum_{t, z} \sum_{j=1}^{3 N_{b}}\left|\left(\phi_{t, z, j}(0), \psi_{i}\right)\right|^{2},
$$

where $\phi_{t, z, j}(0)$ is the $j$-th $2 d$ mode on slice $(t, z)$ in the absence of the magnetic field. In the right panel of Fig. 4 we show what fraction of the valence effect comes from the fact that the nature of the first $3 N_{b}$ modes on all $(z, t)$ slices has changed. The continuum limit has been obtained by assuming that $\frac{\Delta\langle\bar{\psi} \psi(B)\rangle_{L L L}}{\Delta\langle\bar{\psi} \psi(B)\rangle}$ approaches it quadratically in the lattice spacing, which leads to an acceptable $\chi^{2}$ for all values of the magnetic field. The contribution of the $2 d$ LLL modes gets larger and larger as the magnetic field increases, which suggests LLL dominance for large $B$, as expected from effective models. In fact they use the LLL approximation in the limit $q B \gg(\pi T)^{2}$. We show the magnetic field corresponding to $(\pi T)^{2}$ with a vertical line. We conclude that for our largest magnetic field $50 \%$ of the valence effect comes from the LLL in the physical $4 \mathrm{~d}$ case. In the future it will be very interesting to see whether the "sea" effect can also be reproduced in the LLL approximation.

\section{References}

[1] J. O. Andersen, W. R. Naylor and A. Tranberg, Rev. Mod. Phys. 88, 025001 (2016) [arXiv:1411.7176].

[2] I. A. Shovkovy, Lect. Notes Phys. 871, 13 (2013) [arXiv:1207.5081].

[3] G. S. Bali, F. Bruckmann, G. Endrődi, Z. Fodor, S. D. Katz, S. Krieg, A. Schäfer and K. K. Szabó, JHEP 1202, 044 (2012) [arXiv:1111.4956].

[4] M. D'Elia and F. Negro, Phys. Rev. D 83, 114028 (2011) [arXiv:1103.2080].

[5] T. Banks and A. Casher, Nucl. Phys. B 169, 103 (1980).

[6] F. Bruckmann, G. Endrődi and T. G. Kovács, JHEP 1304, 112 (2013) [arXiv:1303.3972].

[7] K. Fukushima, Phys. Rev. D 83, 111501 (2011) [arXiv:1103.4430].

[8] C. N. Leung and S. Y. Wang, Annals Phys. 322, 701 (2007) [hep-ph/0503298].

[9] J. P. Blaizot, E. S. Fraga and L. F. Palhares, Phys. Lett. B 722, 167 (2013) [arXiv:1211.6412].

[10] E. J. Ferrer, V. de la Incera and X. J. Wen, Phys. Rev. D 91, 054006 (2015) [arXiv:1407.3503].

[11] N. Mueller and J. M. Pawlowski, Phys. Rev. D 91, 116010 (2015) [arXiv:1502.08011].

[12] S. Fayazbakhsh and N. Sadooghi, Phys. Rev. D 83, 025026 (2011) [arXiv:1009.6125].

[13] K. Fukushima, K. Hattori, H. U. Yee and Y. Yin, Phys. Rev. D 93, 074028 (2016) [arXiv:1512.03689].

[14] J. Braun, W. A. Mian and S. Rechenberger, Phys. Lett. B 755, 265 (2016) [arXiv:1412.6025].

[15] D. R. Hofstadter, Phys. Rev. B 14, 2239 (1976).

[16] G. Endrődi, PoS LATTICE 2014, 018 (2014) [arXiv:1410.8028].

[17] T. G. Kovács and F. Pittler, Phys. Rev. D 86, 114515 (2012) [arXiv:1208.3475]. 